\title{
Cytotoxic Prenyl and Geranyl Coumarins from the Stem Bark of Casi- miroa edulis
}

\author{
Khun Nay Win Tun ${ }^{1,2}$, Nanik Siti Aminah ${ }^{3, *}$, Alfinda Novi Kristanti ${ }^{3}$, Rico Ramadhan ${ }^{3}$ and \\ Yoshiaki Takaya $^{4}$
}

${ }^{I}$ Natural Science, Faculty of Science and Technology, Universitas Airlangga, Surabaya, Indonesia; ${ }^{2}$ Department of Chemistry, Taunggyi University, Taunggyi, Myanmar; ${ }^{3}$ Department of Chemistry, Faculty of Science and Technology, Universitas Airlangga, Surabaya, Indonesia; ${ }^{4}$ Faculty of Pharmacy, Universitas Meijo, 150 Yagotoyama, Tempaku, Nagoya, 468-8503 Japan

\begin{tabular}{l} 
A R T I C L E H I S T O R Y \\
\hline Received: June 12, 2019 \\
Revised: September 15, 2019 \\
Accepted: October 04, 2019 \\
DOI: \\
10.2174/1570178616666191019121437
\end{tabular}

\begin{abstract}
Phytochemical investigation of the methanolic extract of the stem bark of Casimiroa edulis afforded four coumarins. Various spectroscopic experiments were used to characterize the isolated coumarins. The structures were identified as auraptene (K-1), suberosin (K-2), 5-geranyloxypsoralen (bergamottin) (K-3), and 8-geranyloxypsoralen (K-4), based on the chemical and spectral analysis. Among these compounds, suberosin (K-2) and 5-geranyloxypsoralen (bergamottin) (K-3) were isolated for the first time from this genus, and auraptene (K-1) was isolated from this plant for the first time. Cytotoxicity of pure compound K-4 and sub-fraction MD-3 was evaluated against HeLa and T47D cell lines and moderate activity was found with an $\mathrm{IC}_{50}$ value in the range 17.4 to $72.33 \mu \mathrm{g} / \mathrm{mL}$.
\end{abstract}

Keywords: Casimiroa edulis, coumarins, HeLa, spectroscopic experiments, stem bark, T47D.

\section{INTRODUCTION}

Nature is a good source of potential chemotherapeutic drugs [1]. The isolation process is a key step in discovering new biologically active substances from complex natural extracts [2]. A large number of bioactive compounds are isolated from research studies and screened each year, thus realizing the intrinsic therapeutic potential of natural products, and providing vast resource for further research [3]. Despite significant developments in the extraction and separation techniques, it is still a challenging task to isolate natural products from plants, animals, marine organisms or micro-organisms [4].

Coumarins (2H-1-benzopyran-2-ones) are a class of naturally occurring compounds found in various plants with an extensive pharmacological profile. To date, approximately 1500 coumarin derivatives have been identified from plants [5]. They have been identified as potent anti-inflammatory [6,10], anti-oxidant [7], anti-melanogenic [8], anti-bacterial [9], anti-viral [10], anti-coagulant [11], and cytotoxic agents [12].

A number of coumarins from Casimiroa spp. have been found by several researchers. Phellopterin, isopimpinellin,

*Address correspondence to this author at the Department of Chemistry, Faculty of Science and Technology, Universitas Airlangga; Tel/Fax: +62-

31-5936501; Fax +62-31-5936502; E-mail: nanik-s-a@fst.unair.ac.id imperatorin, xanthotoxol, 8-hydroxy-5-methoxypsoralen, 8-[(6,7-dihydroxy-3,7-dimethyl-2-octen-1-yl)oxy]-5-methoxypsoralen, 8-[(4-hydroxy-3-methyl-2-buten-1-yl)oxy]psoralen, 8-[(6,7-dihydroxy-3,7-dimethyl-2-octen-1-yl)oxy]psoralen, 8-geranyloxypsoralen (K-4), 8-[(4-hydroxy-3-methyl-2-buten1-yl)oxy]-5-methoxypsoralen, are reported to belong to the furanocoumarins, a class of chemical compounds produced by the roots, seeds, and leaves of Casimiroa spp. Another report has shown that simple coumarins (umbelliferone, esculetin, herniarin) are present in the leaves and seeds of Casimiroa spp. Some isolated furanocoumarins and simple coumarins have been reported to show anticoagulant and antimutagenic activity. In addition, the structure modification of some furanocoumarins was also reported [13-20].

This paper outlines the isolation and structure elucidation of compounds K-1 to K-4 (Figs. 1-4) from the stem bark of Casimiroa edulis, as well as the cytotoxicity of sub-fraction MD-3 (section 3.3) and pure compound K-4.

\section{RESULTS AND DISCUSSION}

\subsection{Structure Elucidation}

Consecutive chromatographic purification of the $\mathrm{MeOH}$ soluble fraction of the stem bark of $C$. edulis yielded four compounds. The molecular structures of isolated compounds were identified on the basis of their UV, IR, ${ }^{1} \mathrm{H}$ NMR, ${ }^{13} \mathrm{C}$ NMR, DEPT (Distortionless Enhancement by Polarization 